\title{
EDITORIAL
}

\section{5 years of Panic Disorder research at Maastricht University}

Schruers KRJ. 25 years of Panic Disorder research at Maastricht University. Acta Neuropsychiatrica 2004: 16:55-56. (C) Blackwell Munksgaard 2004

\author{
K. R. J. Schruers \\ Department of Psychiatry and Neuropsychology and Vijverdal \\ Academic Anxiety Center, Maastricht University, Maastricht, \\ the Netherlands \\ Correspondence: K. R. J. Schruers, Department of Psychiatry and \\ Neuropsychology and Vijverdal Academic Anxiety Center, \\ Maastricht University, PO Box 88, 6200 AB Maastricht, the \\ Netherlands. Tel: 0031 433685330; Fax: 0031 433685331; \\ E-mail: koen.schruers@pn.unimaas.nl
}

In 1979, the Academic Anxiety Center was founded by Prof Griez in Maastricht, as a collaboration between Maastricht University and Vijverdal Academic Mental Health Center. From the beginning, focus has been on the exploration of the pathophysiology of panic. As an experimental model to study the phenomenon of panic, the $35 \%$ carbon dioxide inhalation challenge was developed. The validity of this model is now well established and has allowed the study of questions such as the role of the 5-HT (serotonin) system in panic or the relationship between panic and respiratory disorders such as asthma. Other challenge procedures have been introduced as well, including the cholecystokinin (CCK) or pentagastrin challenge, tryptophan depletion, 5-hydroxytryptophan administration or lactate infusion. Over the years, collaboration with a number of international centers of expertise in anxiety disorders has been intense. This has not only contributed to the knowledge about anxiety disorders but has also spawned the first international research master program: the Master in Affective Neuroscience, awarded jointly by the Universities of Maastricht, the Netherlands, and Florence, Italy.

The present special edition of the Acta Neuropsychiatrica is part of the celebration of 25 years of panic research in Maastricht and of an equally longstanding international collaboration. In this issue, all papers are focused around panic disorder. Some are from our own team, others are contributions by eminent researchers we have had the honour to work with.

Most have a review character but there are also some original contributions.
In the first paper, Perna et al. explore the panic respiration connection. They convincingly defend the viewpoint that panic is the expression of the abnormal regulation of respiratory functions or, more generally, of homeostasis of the internal organism milieu. Results of genetic research into panic have been modest up to present. Van West and Claes provide a critical overview of the available genetic studies and suggest directions for future research. In particular the combination between genetics and biochemical challenge methods, to define more homogeneous samples, is promising. This could include the manipulation of 5-HT availability to the brain. One such method, tryptophan depletion, and its use in panic disorder is described in detail by Pyle et al. It appears that 5-HT is mainly important in the regulation of panic and inherited deficits in 5-HT metabolism could be responsible for increased susceptibility to panic. Cholecystokinin is one of the most ubiquitous neurotransmitters in the brain but is nevertheless often overlooked. However, panic provocation with CCK is well established. Bourin and Dailly review the role of CCK in anxiety and panic, starting from animal models and leading to human pharmacological studies. An emerging field is the role of the immune system in anxiety disorders. Van Duinen et al. provide a systematic review of neuro-immunological findings in PD. They also reach the conclusion that future studies should preferably include experimental laboratory panic provocation. Two original contributions are included in this issue. The first describes the symptom profiles of panic attacks, as measured in real life and compared to the laboratory situation. The other is about 
the ability of ethanol to attenuate experimental panic. The focus in this issue is on the neurobiological aspects of the phenomenon panic. To remind us that learning theory, which has evident links with neurobiology, and cognitive theory have also contributed considerably to our knowledge about panic, a brief overview of new insights in learning and cognitive theory concludes this issue. 